\title{
The factorization and monotonicity method for the defect in an open periodic waveguide
}

\author{
Takashi FURUYA
}

\begin{abstract}
In this paper, we consider the inverse scattering problem to reconstruct the defect in an open periodic waveguide from near field data. Our first aim is to mention that there is a mistake in the factorization method of Lechleiter [13]. By this we can not apply it to solve this inverse problem. Our second aim is to give ways to understand the defect from inside (Theorem 1.1) and outside (Theorem 1.2) based on the monotonicity method. Finally, we give numerical examples based on Theorem 1.1.
\end{abstract}

\section{Introduction}

In this paper, we consider the inverse scattering problem to reconstruct the defect in an open periodic waveguide from near field data. The contributions of this paper are followings.

- We mention that there is a mistake in $\left(F_{\#^{-}}\right)$factorization method of Lechleiter [13] by giving a counterexample, and revise the proof of the factorization method.

- We give the reconstruction method based on the monotonicity method. The unknown defect are understood from inside (Theorem 1.1) and outside (Theorem 1.2)

By the mistake we will understand that transmission eigenvale is needed in the factorization method for inverse medium scattering problem. Furthermore, we can not apply it to inverse problem of an open periodic waveguide we will consider here. In order to solve the inverse problem of our case, we consider the monotonicity method. The feature of this method is to understand the inclusion relation of an unknown defect and an artificial domain by comparing the data operator with some operator corresponding to an artificial one. For recent works of the monotonicity method, we refer to [2, 3, 4, 15, 6, 7, 12].

We begin with formulation of the scattering problem. Let $k>0$ be the wave number, and let $\mathbb{R}_{+}^{2}:=\mathbb{R} \times(0, \infty)$ be the upper half plane, and let $W:=\mathbb{R} \times(0, h)$ be the waveguide in $\mathbb{R}_{+}^{2}$. We denote by $\Gamma_{a}:=\mathbb{R} \times\{a\}$ for 
$a>0$. Let $n \in L^{\infty}\left(\mathbb{R}_{+}^{2}\right)$ be real value, $2 \pi$-periodic with respect to $x_{1}$ (that is, $n\left(x_{1}+2 \pi, x_{2}\right)=n\left(x_{1}, x_{2}\right)$ for all $\left.x=\left(x_{1}, x_{2}\right) \in \mathbb{R}_{+}^{2}\right)$, and equal to one for $x_{2}>h$. We assume that there exists a constant $n_{\max }>0$ and $n_{\text {min }}>0$ such that $n_{\text {min }} \leq n \leq n_{\max }$ in $\mathbb{R}_{+}^{2}$. Let $q \in L^{\infty}\left(\mathbb{R}_{+}^{2}\right)$ be real value with the compact support in $W$. We denote by $Q:=\operatorname{supp} q$. Assume that $\mathbb{R}^{2} \backslash \bar{Q}$ is connected. First of all we consider the following direct scattering problem: For fixed $y \in \mathbb{R}_{+}^{2} \backslash \bar{W}$, determine the scattered field $u^{s} \in H_{l o c}^{1}\left(\mathbb{R}_{+}^{2}\right)$ such that

$$
\begin{gathered}
\Delta u^{s}+k^{2}(1+q) n u^{s}=-k^{2} q n u^{i}(\cdot, y) \text { in } \mathbb{R}_{+}^{2}, \\
u^{s}=0 \text { on } \Gamma_{0},
\end{gathered}
$$

where the incident field $u^{i}$ is given by $u^{i}(x, y)=\overline{G_{n}(x, y)}$, where $G_{n}$ is the Dirichlet Green's function in the upper half plane $\mathbb{R}_{+}^{2}$ for $\Delta+k^{2} n$, that is,

$$
G_{n}(x, y):=G(x, y)+\tilde{u}^{s}(x, y),
$$

where $G(x, y):=\Phi_{k}(x, y)-\Phi_{k}\left(x, y^{*}\right)$ is the Dirichlet Green's function for $\Delta+k^{2}$, and $y^{*}=\left(y_{1},-y_{2}\right)$ is the reflected point of $y$ at $\mathbb{R} \times\{0\}$. Here, $\Phi_{k}(x, y)$ is the fundamental solution to Helmholtz equation in $\mathbb{R}^{2}$, that is,

$$
\Phi_{k}(x, y):=\frac{i}{4} H_{0}^{(1)}(k|x-y|), x \neq y .
$$

$\tilde{u}^{s}$ is the scattered field of the unperturbed problem by the incident field $G(x, y)$, that is, $\tilde{u}^{s}$ vanishes for $x_{2}=0$ and solves

$$
\Delta \tilde{u}^{s}+k^{2} n \tilde{u}^{s}=k^{2}(1-n) G(\cdot, y) \text { in } \mathbb{R}_{+}^{2} .
$$

If we impose a suitable radiation condition introduced by Kirsch and Lechleiter [11, the unperturbed solution $\tilde{u}^{s}$ is uniquely determined. Later, we will explain the exact definition of this radiation condition (see Definition 2.4). Furthermore, with the same radiation condition and an additional assumption (see Assumption 2.7) the well-posedness of the problem (1.1)-(1.2) was show in [1].

By the well-posedness of this perturbed scattering problem, we are able to consider the inverse problem of determining the support of $q$ from measured scattered field $u^{s}$ by the incident field $u^{i}$. Let $M:=\left\{\left(x_{1}, m\right): a<\right.$ $\left.x_{1}<b\right\}$ for $a<b$ and $m>h$, and $Q:=\operatorname{supp} q$. With the scattered field $u^{s}$, we define the near field operator $N: L^{2}(M) \rightarrow L^{2}(M)$ by

$$
N g(x):=\int_{M} u^{s}(x, y) g(y) d s(y), x \in M
$$


The inverse problem we consider in this paper is to determine support $Q$ of $q$ from the scattered field $u^{s}(x, y)$ for all $x$ and $y$ in $M$ with one $k>0$. In other words, given the near field operator $N$, determine $Q$.

Our aim in this paper is to revise the factorization method (see section 3 ) and to provide the following two theorems.

Theorem 1.1. Let $B \subset \mathbb{R}^{2}$ be a bounded open set. Let Assumption hold, and assume that there exists $q_{\min }>0$ such that $q \geq q_{\min }$ a.e. in $Q$. Then for $0<\alpha<k^{2} n_{\min } q_{\min }$,

$$
B \subset Q \quad \Longleftrightarrow \quad \alpha H_{B}^{*} H_{B} \leq_{\text {fin }} \operatorname{Re} N
$$

where the operator $H_{B}: L^{2}(M) \rightarrow L^{2}(B)$ is given by

$$
H_{B} g(x):=\int_{M} \overline{G_{n}(x, y)} g(y) d s(y), x \in B,
$$

and the inequality on the right hand side in (1.7) denotes that $\operatorname{Re} N-\alpha H_{B}^{*} H_{B}$ has only finitely many negative eigenvalues, and the real part of an operator $A$ is self-adjoint operators given by $\operatorname{Re}(A):=\frac{1}{2}\left(A+A^{*}\right)$.

Theorem 1.2. Let $B \subset \mathbb{R}^{2}$ be a bounded open set. Let Assumption hold, and assume that there exists $q_{\min }>0$ and $q_{\max }>0$ such that $q_{\min } \leq q \leq q_{\max }$ a.e. in $Q$. Then for $\alpha>k^{2} n_{\max } q_{\max }$,

$$
Q \subset B \quad \Longleftrightarrow \quad \operatorname{Re} N \leq_{\text {fin }} \alpha H_{B}^{*} H_{B},
$$

We understand whether an artificial domain $B$ is contained in $Q$ or not in Theorem 1.1, and $B$ contain $Q$ in Theorem 1.2, respectively. Then, by preparing a lot of known domain $B$ and for each $B$ checking (1.7) or 1.9) we can reconstruct the shape and location of unknown $Q$.

This paper is organized as follows. In Section 2, we recall a radiation condition introduced in [11, and the well-posedness of the problem (1.1)(1.2). In Section 3, we mention the exact functional analytic theorem in the factorization method (Theorem 2.15 in [9]), and mention where there is a mistake in one of Lechleiter (Theorem 2.1 in [13]) by giving an counterexample. In Section 4, we consider several factorization of the near field operator $N$, and we mention that there is a difficulty to apply the factorization method due to the mistake of Lechleiter. However, the properties of its factorization discussed in Section 4 will be useful when we show Theorems 1.1 and 1.2. In Sections 5 and 6, we prove Theorems 1.1 and 1.2, respectively. Finally in Section 7, numerical examples based on Theorem 1.1 are given. 


\section{A radiation condition}

In Section 2, we recall a radiation condition introduced in [11. Let $f \in$ $L^{2}\left(\mathbb{R}_{+}^{2}\right)$ have the compact support in $W$. First, we consider the following direct problem: Determine the scattered field $u \in H_{l o c}^{1}\left(\mathbb{R}_{+}^{2}\right)$ such that

$$
\begin{gathered}
\Delta u+k^{2} n u=f \text { in } \mathbb{R}_{+}^{2}, \\
u=0 \text { on } \Gamma_{0} .
\end{gathered}
$$

(2.1) is understood in the variational sense, that is,

$$
\int_{\mathbb{R}_{+}^{2}}\left[\nabla u \cdot \nabla \bar{\varphi}-k^{2} n u \bar{\varphi}\right] d x=-\int_{W} f \bar{\varphi} d x,
$$

for all $\varphi \in H^{1}\left(\mathbb{R}_{+}^{2}\right)$, with compact support. In such a problem, it is natural to impose the upward propagating radiation condition, that is, $u(\cdot, h) \in L^{\infty}(\mathbb{R})$ and

$$
u(x)=2 \int_{\Gamma_{h}} u(y) \frac{\partial \Phi_{k}(x, y)}{\partial y_{2}} d s(y)=0, x_{2}>h .
$$

However, even with this condition we can not expect the uniqueness of this problem. (see Example 2.3 of [11.) In order to introduce a suitable radiation condition, Kirsch and Lechleiter discussed limiting absorption solution of this problem, that is, the limit of the solution $u_{\epsilon}$ of $\Delta u_{\epsilon}+(k+i \epsilon)^{2} n u_{\epsilon}=f$ as $\epsilon \rightarrow 0$. For the details of an introduction of this radiation condition, we refer to [10, 11].

Let us prepare for the exact definition of the radiation condition. We denote by $C_{R}:=(0,2 \pi) \times(0, R)$ for $R \in(0, \infty]$. The function $u \in H^{1}\left(C_{R}\right)$ is called $\alpha$-quasi periodic if $u\left(2 \pi, x_{2}\right)=e^{2 \pi i \alpha} u\left(0, x_{2}\right)$. We denote by $H_{\alpha}^{1}\left(C_{R}\right)$ the subspace of the $\alpha$-quasi periodic function in $H^{1}\left(C_{R}\right)$, and $H_{\alpha, l o c}^{1}\left(C_{\infty}\right):=$ $\left\{u \in H_{l o c}^{1}\left(C_{\infty}\right):\left.u\right|_{C_{R}} \in H_{\alpha}^{1}\left(C_{R}\right)\right.$ for all $\left.\mathrm{R}>0\right\}$. Then, we consider the following problem, which arises from taking the quasi-periodic Floquet Bloch transform (see, e.g., [14].) in (2.1)-2.2): For $\alpha \in[-1 / 2,1 / 2]$, determine $u_{\alpha} \in H_{\alpha, l o c}^{1}\left(C_{\infty}\right)$ such that

$$
\begin{gathered}
\Delta u_{\alpha}+k^{2} n u_{\alpha}=f_{\alpha} \text { in } C_{\infty} . \\
u_{\alpha}=0 \text { on }(0,2 \pi) \times\{0\} .
\end{gathered}
$$

Here, it is a natural to impose the Rayleigh expansion of the form

$$
u_{\alpha}(x)=\sum_{n \in \mathbb{Z}} u_{n}(\alpha) e^{i n x_{1}+i \sqrt{k^{2}-(n+\alpha)^{2}}\left(x_{2}-h\right)}, x_{2}>h,
$$


where $u_{n}(\alpha):=(2 \pi)^{-1} \int_{0}^{2 \pi} u_{\alpha}\left(x_{1}, h\right) e^{-i n x_{1}} d x_{1}$ are the Fourier coefficients of $u_{\alpha}(\cdot, h)$, and $\sqrt{k^{2}-(n+\alpha)^{2}}=i \sqrt{(n+\alpha)^{2}-k^{2}}$ if $n+\alpha>k$. But even with this expansion the uniqueness of this problem fails for some $\alpha \in$ $[-1 / 2,1 / 2]$. We call $\alpha$ exceptional values if there exists non-trivial solutions $u_{\alpha} \in H_{\alpha, l o c}^{1}\left(C_{\infty}\right)$ of $2.5-(2.7)$. We set $A_{k}:=\{\alpha \in[-1 / 2,1 / 2]: \exists l \in$ $\mathbb{Z}$ s.t. $|\alpha+l|=k\}$, and make the following assumption:

Assumption 2.1. For every $\alpha \in A_{k}$ the solution of $u_{\alpha} \in H_{\alpha, l o c}^{1}\left(C_{\infty}\right)$ of (2.5)-2.7) has to be zero.

The following properties of exceptional values was shown in [11].

Lemma 2.2. Let Assumption 2.1 hold. Then, there exists only finitely many exceptional values $\alpha \in[-1 / 2,1 / 2]$. Furthermore, if $\alpha$ is an exceptional value, then so is $-\alpha$. Therefore, the set of exceptional values can be described by $\left\{\alpha_{j}: j \in J\right\}$ where some $J \subset \mathbb{Z}$ is finite and $\alpha_{-j}=-\alpha_{j}$ for $j \in J$. For each exceptional value $\alpha_{j}$ we define

$$
X_{j}:=\left\{\phi \in H_{\alpha_{j}, l o c}^{1}\left(C_{\infty}\right): \begin{array}{c}
\Delta \phi+k^{2} n \phi=0 \text { in } C_{\infty}, \quad \phi=0 \text { for } x_{2}=0 \\
\phi \text { satisfies the Rayleigh expansion } 2.7
\end{array}\right\}
$$

Then, $X_{j}$ are finite dimensional. We set $m_{j}=\operatorname{dim} X_{j}$. Furthermore, $\phi \in X_{j}$ is evanescent, that is, there exists $c>0$ and $\delta>0$ such that $|\phi(x)|,|\nabla \phi(x)| \leq c e^{-\delta\left|x_{2}\right|}$ for all $x \in C_{\infty}$.

Next, we consider the following eigenvalue problem in $X_{j}$ : Determine $d \in \mathbb{R}$ and $\phi \in X_{j}$ such that

$$
-i \int_{C_{\infty}} \frac{\partial \phi}{\partial x_{1}} \bar{\psi} d x=d k \int_{C_{\infty}} n \phi \bar{\psi} d x
$$

for all $\psi \in X_{j}$. We denote by the eigenvalues $d_{l, j}$ and eigenfunction $\phi_{l, j}$ of this problem, that is,

$$
-i \int_{C_{\infty}} \frac{\partial \phi_{l, j}}{\partial x_{1}} \bar{\psi} d x=d_{l, j} k \int_{C_{\infty}} n \phi_{l, j} \bar{\psi} d x
$$

for every $l=1, \ldots, m_{j}$ and $j \in J$. We normalize the eigenfunction $\left\{\phi_{l, j}: l=\right.$ $\left.1, \ldots, m_{j}\right\}$ such that

$$
k \int_{C_{\infty}} n \phi_{l, j} \overline{\phi_{l^{\prime}, j}} d x=\delta_{l, l^{\prime}}
$$

for all $l, l^{\prime}$. We will assume that the wave number $k>0$ is regular in the following sense. 
Definition 2.3. $k>0$ is regular if $d_{l, j} \neq 0$ for all $l=1, \ldots m_{j}$ and $j \in J$.

Now we are ready to define the radiation condition.

Definition 2.4. Let Assumptions 2.1 hold, and let $k>0$ be regular in the sense of Definition 2.3. We set

$$
\psi^{ \pm}\left(x_{1}\right):=\frac{1}{2}\left[1 \pm \frac{2}{\pi} \int_{0}^{x_{1} / 2} \frac{\sin t}{t} d t\right], x_{1} \in \mathbb{R} .
$$

Then, $u \in H_{l o c}^{1}\left(\mathbb{R}_{+}^{2}\right)$ satisfies the radiation condition if $u$ satisfies the upward propagating radiation condition (2.4), and has a decomposition in the form $u=u^{(1)}+u^{(2)}$ where $\left.u^{(1)}\right|_{\mathbb{R} \times(0, R)} \in H^{1}(\mathbb{R} \times(0, R))$ for all $R>0$, and $u^{(2)} \in L^{\infty}\left(\mathbb{R}_{+}^{2}\right)$ has the following form

$$
u^{(2)}(x)=\psi^{+}\left(x_{1}\right) \sum_{j \in J} \sum_{d_{l, j}>0} a_{l, j} \phi_{l, j}(x)+\psi^{-}\left(x_{1}\right) \sum_{j \in J} \sum_{d_{l, j}<0} a_{l, j} \phi_{l, j}(x)
$$

where some $a_{l, j} \in \mathbb{C}$, and $\left\{d_{l, j}, \phi_{l, j}: l=1, \ldots, m_{j}\right\}$ are normalized eigenvalues and eigenfunctions of the problem (2.8).

Remark 2.5. It is obvious that we can replace $\psi^{+}$by any smooth functions $\tilde{\psi}^{ \pm}$with $\tilde{\psi}^{+}\left(x_{1}\right)=1+\mathcal{O}\left(1 / x_{1}\right)$ as $x_{1} \rightarrow \infty$ and $\tilde{\psi}^{+}\left(x_{1}\right)=\mathcal{O}\left(1 / x_{1}\right)$ as $x_{1} \rightarrow-\infty$ and $\frac{d}{d x_{1}} \tilde{\psi}^{+}\left(x_{1}\right) \rightarrow 0$ as $\left|x_{1}\right| \rightarrow \infty$ (and analogously for $\psi^{-}$).

The following was shown in Theorems 2.2,6.6, and 6.8 of [11].

Theorem 2.6. For every $f \in L^{2}\left(\mathbb{R}_{+}^{2}\right)$ with the compact support in $W$, there exists a unique solution $u_{k+i \epsilon} \in H^{1}\left(\mathbb{R}_{+}^{2}\right)$ of the problem (2.1)-(2.2) replacing $k$ by $k+i \epsilon$. Furthermore, $u_{k+i \epsilon}$ converge as $\epsilon \rightarrow+0$ in $H_{l o c}^{1}\left(\mathbb{R}_{+}^{2}\right)$ to some $u \in H_{\text {loc }}^{1}\left(\mathbb{R}_{+}^{2}\right)$ which satisfy (2.1)-(2.2) and the radiation condition in the sense of Definition 2.4. Furthermore, the solution $u$ of this problem is uniquely determined.

Furthermore, with the same radiation condition and the following additional assumption, the well-posedness of the perturbed scattering problem of (2.1) -2.2 was show in [1].

Assumption 2.7. We assume that $k^{2}$ is not the point spectrum of $\frac{1}{(1+q) n} \Delta$ in $H_{0}^{1}\left(\mathbb{R}_{+}^{2}\right)$, that is, evey $v \in H^{1}\left(\mathbb{R}_{+}^{2}\right)$ which satisfies

$$
\begin{gathered}
\Delta v+k^{2}(1+q) n v=0 \text { in } \mathbb{R}_{+}^{2}, \\
v=0 \text { on } \Gamma_{0},
\end{gathered}
$$

has to vanishes for $x_{2}>0$. 
Theorem 2.8. Let Assumption 2.7 hold and let $f \in L^{2}\left(\mathbb{R}_{+}^{2}\right)$ such that $\operatorname{supp} f=Q$. Then, there exists a unique solution $u \in H_{\text {loc }}^{1}\left(\mathbb{R}_{+}^{2}\right)$ such that

$$
\begin{gathered}
\Delta u+k^{2}(1+q) n u=f \text { in } \mathbb{R}_{+}^{2}, \\
u=0 \text { on } \Gamma_{0},
\end{gathered}
$$

and $u$ satisfies the radiation condition in the sense of Definition 2.4.

By Theorem 2.8, the well-posedness of the perturbed scattering problem (1.1) - 1.2 with the radiation condition follows. Then, we are able to consider the inverse problem of determining the support of $q$ from measured scattered field $u^{s}$ by the incident field $u^{i}(x, y)=\overline{G_{n}(x, y)}$. In the following sections we will discuss the inverse problem.

\section{The factorization method}

In Section 3, we mention the exact functional analytic theorem in $\left(F_{\#^{-}}\right)$ factorization method. The following functional analytic theorem is given by the almost same argument in Theorem 2.15 of [9].

Theorem 3.1. Let $X \subset U \subset X^{*}$ be a Gelfand triple with a Hilbert space $U$ and a reflexive Banach space $X$ such that the imbedding is dense. Furthermore, let $Y$ be a second Hilbert space and let $F: Y \rightarrow Y, G: X \rightarrow Y$, $T: X^{*} \rightarrow X$ be linear bounded operators such that

$$
F=G T G^{*} \text {. }
$$

We make the following assumptions:

(1) $G$ is compact with dense range in $Y$.

(2) There exists $t \in[0,2 \pi]$ such that $\operatorname{Re}\left(\mathrm{e}^{i t} T\right)$ has the form $\operatorname{Re}\left(\mathrm{e}^{i t} T\right)=C+$ $K$ with some compact operator $K$ and some self-adjoint and positive coercive operator $C$, i.e., there exists $c>0$ such that

$$
\langle\varphi, C \varphi\rangle \geq c\|\varphi\|^{2} \text { for all } \varphi \in X^{*} .
$$

(3) $\operatorname{Im}\langle\varphi, T \varphi\rangle>0$ for all $\varphi \in \overline{\operatorname{Ran}\left(G^{*}\right)}$ with $\varphi \neq 0$.

Then, the operator $F_{\#}:=\left|\operatorname{Re}\left(\mathrm{e}^{i t} F\right)\right|+\operatorname{Im} F$ is non-negative, and the ranges of $G: X \rightarrow Y$ and $F_{\#}^{1 / 2}: Y \rightarrow Y$ coincide with each other, that is, we have the following range identity;

$$
\operatorname{Ran}(G)=\operatorname{Ran}\left(F_{\#}^{1 / 2}\right) .
$$


Here, the real part and the imaginary part of an operator $A$ are selfadjoint operators given by

$$
\operatorname{Re}(A)=\frac{A+A^{*}}{2} \text { and } \operatorname{Im}(A)=\frac{A-A^{*}}{2 i} .
$$

Remark 3.2. Here, we will mention a mistake of Theorem 2.1 in 13 . It was introduced in order to avoid that $k^{2}$ is not a transmission eigenvalue corresponding to the unknown medium. To realize it, we replaced the assumptions (3) by the injectivity of $T$. However, its condition is not enough to obtain the range identity (3.3). The following matrixes are its counterexample in which the strictly positivity of $\operatorname{Im} T$ is missing and the range identity (3.3) fails.

$$
\begin{gathered}
\left(\begin{array}{llll}
1 & 0 & 0 & 0 \\
0 & 1 & 0 & 0
\end{array}\right)\left(\begin{array}{llll}
0 & 0 & 0 & 1 \\
0 & 0 & 1 & 0 \\
0 & 1 & 0 & 0 \\
1 & 0 & 0 & 0
\end{array}\right)\left(\begin{array}{ll}
1 & 0 \\
0 & 1 \\
0 & 0 \\
0 & 0
\end{array}\right)=\left(\begin{array}{ll}
0 & 0 \\
0 & 0
\end{array}\right), \\
\operatorname{Ran}\left(\begin{array}{llll}
1 & 0 & 0 & 0 \\
0 & 1 & 0 & 0
\end{array}\right) \neq \operatorname{Ran}\left(\begin{array}{ll}
0 & 0 \\
0 & 0
\end{array}\right) .
\end{gathered}
$$

From this counterexample one can not expect the range identity without $\operatorname{Im} T>0$. Therefore, in the factorization method for inverse medium scattering problem, we have to assume that $k^{2}$ is not a transmission eigenvalue corresponding to the unknown medium in order to have the strictly positivity of $\operatorname{Im} T$.

In this section, we will prove Theorem 3.1 based on Theorem 2.15. We remark that in Theorem 2.15 of [9] we assumed that $\operatorname{Im} T$ is compact, while in Theorem 3.1 of this section we will not assume its compactness. (The operator $\operatorname{Im} T$ is not compact in the case of inverse medium scattering problem with complex valued contrast function, See Theorem 4.5 of [9].) Before the proof of Theorem 3.1, we will show the following lemma.

Lemma 3.3. Let $X$ be a Hlbert space, and let $T: X \rightarrow X$ be a linear bounded, and let $K: X \rightarrow X$ be a linear bounded injective. We assume that

$$
\operatorname{Ran}(T) \text { is closed subspace in } X \text {, and } \operatorname{dimKer}(T)<\infty \text {. }
$$

Then, there is a constant $C>0$ such that

$$
\|u\|_{X}^{2} \leq C\left(\|T u\|_{X}^{2}+\|K u\|_{X}^{2}\right) \quad \text { for all } u \in X .
$$


Proof of Lemma 3.3. Assume that on contrary for any $C>0$, there exists a $u_{c} \in X$ such that

$$
\left\|u_{c}\right\|_{X}^{2}>C\left(\left\|T u_{c}\right\|_{X}^{2}+\left\|K u_{c}\right\|_{X}^{2}\right)
$$

Then, we can choose a sequence $\left\{u_{m}\right\} \subset X$ with $\left\|u_{m}\right\|^{2}=1$ such that $\left\|T u_{m}\right\|^{2}+\left\|K u_{m}\right\|^{2}$ converge to zero as $m \rightarrow \infty$. Since $\operatorname{Ker}(T)$ is finite dimensional subspace in $X$, there exists an orthogonal complement $\operatorname{Ker}(T)^{\perp}$ of $\operatorname{Ker}(T)$ such that $X=\operatorname{Ker}(T) \oplus \operatorname{Ker}(T)^{\perp}$. Since $\operatorname{Ker}(T)^{\perp}$ and $\operatorname{Ran}(T)$ is closed subspaces in $X$, the restrict operator $\left.T\right|_{\operatorname{Ker}(T)^{\perp}}$ is injective and surjective from the Banach space $\operatorname{Ker}(T)^{\perp}$ to the Banach space $\operatorname{Ran}(T)$. Then by the closed graph theorem, $\left.T\right|_{\operatorname{Ker}(T)^{\perp}}$ is invertible bounded, which implies that there is a constant $C>0$ such that

$$
\left\|P u_{m}\right\|^{2} \leq C\left\|\left.T\right|_{\operatorname{Ker}(T)^{\perp}} P u_{m}\right\|^{2}=C\left\|T u_{m}\right\|^{2} .
$$

Since $K$ is injective and $\operatorname{Ker}(T)$ is finite dimensional subspace in $X$, there is a constant $C>0$ such that

$$
\|v\| \leq C\|K v\|^{2} \quad \text { for all } v \in \operatorname{Ker}(T) .
$$

(If not, we can take a sequence $\left\{v_{m}\right\} \subset \operatorname{Ker}(T)$ with $\left\|v_{m}\right\|=1$ and $\left\|K v_{m}\right\| \rightarrow$ 0 . Since $\operatorname{Ker}(T)$ is finite dimensional subspace, there exists $v_{0} \in \operatorname{Ker}(T)$ such that $v_{m} \rightarrow v_{0}$ and $K v_{m} \rightarrow K v_{0}$ as $m \rightarrow \infty$, which implies that $v_{0}=0$ by the injectivity of $K$. This contradicts with $\left\|v_{m}\right\|=1$.)

Then, there is a constant $C>0$ such that

$$
\begin{aligned}
\left\|(I-P) u_{m}\right\|^{2} & \leq C\left\|K(I-P) u_{m}\right\|^{2} \leq 2\left(C\left\|K u_{m}\right\|^{2}+\left\|K P u_{m}\right\|^{2}\right) \\
& \leq 2 C\left(\left\|K u_{m}\right\|^{2}+\|K\|^{2}\left\|P u_{m}\right\|^{2}\right) .
\end{aligned}
$$

Therefore, by 3.10 and 3.12 there exists a constant $C^{\prime}>0$ such that

$$
1=\left\|P u_{m}\right\|^{2}+\left\|(I-P) u_{m}\right\|^{2} \leq C^{\prime}\left(\left\|T u_{m}\right\|^{2}+\left\|K u_{m}\right\|^{2}\right) .
$$

As $m \rightarrow \infty$ the right hand side of above inequality converges to zero, which contradicts.

We will show Theorem 3.1.

Proof of Theorem 3.1. By the same argument of Part A (Reduction) in the proof of Theorem $2.15([9]$, we can restrict ourselves to the case $X=U$ and $C=I$. Furthermore, we can also restrict ourselves to the case $G$ is 
injective. Indeed, let $P: U \rightarrow \overline{\operatorname{Ran}\left(G^{*}\right)}$ be the orthogonal projection onto $\hat{U}:=\overline{\operatorname{Ran}\left(G^{*}\right)} \subset U$. Then, $P G^{*}=G^{*}$ and $G=G P$. By this, we can have the factorization of the form

$$
F=G P T P G^{*}=\hat{G} \hat{T} \hat{G}^{*}
$$

where $\left.\hat{G}\right|_{\hat{U}}: \hat{U} \rightarrow Y$ and $\hat{T}=\left.P T\right|_{\hat{U}}: \hat{U} \rightarrow \hat{U}$. Therefore, all of assumptions (1)-(3) are satisfied. (We remark that $\hat{T}$ is not injective even if $T$ is injective, which leads to error in Theorem 2.1 of [13].)

By the same argument in Part B, C, and D in the proof of Theorem 2.15 ([9]), we can show that

$$
F_{\#}=G T_{\#} \hat{G}^{*}
$$

where $T_{\#}=\operatorname{Re}\left(\mathrm{e}^{i t} T\right) D+\operatorname{Im} T$ and $D$ is an isomorphism from $U$ onto itself. It was shown that the operator $T_{\#}$ is non-negative on $U$ in the proof. By applying the inequality (4.5) of [8] to the non-negative operators $\operatorname{Re}\left(\mathrm{e}^{i t} T\right) D$ and $\operatorname{Im} T$, there is a constant $C>0$ such that

$$
\begin{aligned}
\left\langle\varphi, T_{\#} \varphi\right\rangle & =\left\langle\varphi, \operatorname{Re}\left(\mathrm{e}^{i t} T\right) D \varphi\right\rangle+\langle\varphi, \operatorname{Im} T \varphi\rangle \\
& \geq C\left(\left\|\operatorname{Re}\left(\mathrm{e}^{i t} T\right) D \varphi\right\|^{2}+\|\operatorname{Im} T \varphi\|^{2}\right) \quad \text { for all } \varphi \in U .
\end{aligned}
$$

By assumption (2) Re( $\left(\mathrm{e}^{i t} T\right) D$ is a Fredholm operator, and by assumption (3) $\operatorname{Im} T$ is injective. Therefore by applying Lemma 3.3 to our operators, there is a constant $C>0$ such that

$$
C\left(\left\|\operatorname{Re}\left(\mathrm{e}^{i t} T\right) D \varphi\right\|+\|\operatorname{Im} T \varphi\|\right) \geq\|\varphi\| \quad \text { for all } \varphi \in U
$$

which implies that the operator $T_{\#}: U \rightarrow U$ is positive coercive. Since we can write

$$
F_{\#}=F_{\#}^{1 / 2}\left(F_{\#}^{1 / 2}\right)^{*}=G T_{\#} G^{*}
$$

then by applying Theorem 1.21 of [9], we have the range of $F_{\#}^{1 / 2}$ and $G$ coincide. We have shown Theorem 3.1.

\section{A factorization of the near field operator}

In Section 4, we discuss a factorization of the near field operator $N$. We define the operator $L: L^{2}(Q) \rightarrow L^{2}(M)$ by $L f:=\left.v\right|_{M}$ where $v$ satisfies the radiation condition in the sense of Definition 2.4 and

$$
\Delta v+k^{2}(1+q) n v=-k^{2} \frac{n q}{\sqrt{|n q|}} f, \text { in } \mathbb{R}_{+}^{2},
$$




$$
v=0 \text { on } \mathbb{R} \times\{0\} .
$$

We define $H: L^{2}(M) \rightarrow L^{2}(Q)$ by

$$
H g(x):=\sqrt{|n(x) q(x)|} \int_{M} \overline{G_{n}(x, y)} g(y) d s(y), x \in Q .
$$

Then, by these definition we have $N=L H$. In order to make a symmetricity of the factorization of the near field operator $N$, we will show the following symmetricity of the Green function $G_{n}$.

\section{Lemma 4.1.}

$$
G_{n}(x, y)=G_{n}(y, x), x \neq y .
$$

Proof of Lemma 4.1. We take a small $\eta>0$ such that $B_{2 \eta}(x) \cap B_{2 \eta}(y)=$ $\emptyset$ where $B_{\epsilon}(z) \subset \mathbb{R}^{2}$ is some open ball with center $z$ and radius $\epsilon>0$. We recall that $G_{n}(z, y)=G(z, y)+\tilde{u}^{s}(z, y)$ where $G(z, y)=\Phi_{k}(z, y)-\Phi_{k}\left(z, y^{*}\right)$ and $\tilde{u}^{s}(z, y)$ is a radiating solution of the problem $(1.5)$ such that $\tilde{u}^{s}(z, y)=0$ for $z_{2}=0$. In Introduction of [11] $\tilde{u}^{s}$ is given by $\tilde{u}^{s}(z, y)=u(z, y)-\chi(\mid z-$ $y \mid) G(z, y)$ where $\chi \in C^{\infty}\left(\mathbb{R}_{+}\right)$satisfying $\chi(t)=0$ for $0 \leq t \leq \eta / 2$ and $\chi(t)=1$ for $t \geq \eta$, and $u$ is a radiating solution such that $u=0$ on $\mathbb{R} \times\{0\}$ and

$$
\begin{gathered}
\Delta u+k^{2} n u=f(\cdot, y) \text { in } \mathbb{R}_{+}^{2}, \\
u=0 \text { on } \mathbb{R} \times\{0\},
\end{gathered}
$$

where

$f(\cdot, y):=\left[k^{2}(1-n)(1-\chi(|\cdot-y|))+\Delta \chi(|\cdot-y|)\right] G(\cdot, y)+2 \nabla \chi(|\cdot-y|) \cdot \nabla G(\cdot, y)$.

Then, we have $G_{n}(z, y)=u(z, y)+(1-\chi(|z-y|)) G(z, y)$. By Theorem 2.6 we can take an solution $u_{\epsilon} \in H^{1}\left(\mathbb{R}_{+}^{2}\right)$ of the problem 4.5 4.6 replacing $k$ by $(k+i \epsilon)$ satisfying $u_{\epsilon}$ converges as $\epsilon \rightarrow+0$ in $H_{l o c}^{1}\left(\mathbb{R}_{+}^{2}\right)$ to $u$. We set $G_{n, \epsilon}(z, y):=u_{\epsilon}(z, y)+(1-\chi(|z-y|)) G(z, y)$, and $G_{n, \epsilon}(z, y)$ converges as $\epsilon \rightarrow+0$ to $G(z, y)$ pointwise for $z \in \mathbb{R}_{+}^{2}$. By the simple calculation, we have

$$
\left[\Delta_{z}+(k+i \epsilon)^{2} n(z)\right] G_{n, \epsilon}(z, y)=-\delta(z, y)+\left(2 k \epsilon i-\epsilon^{2}\right) n(z)(1-\chi(|z-y|)) G(z, y) \text {. }
$$

Let $r>0$ be large enough such that $x, y \in B_{r}(0)$. By Green's second 
theorem in $B_{r}(0) \cap \mathbb{R}_{+}^{2}$ we have

$$
\begin{aligned}
& -G_{n, \epsilon}(y, x)+\left(2 k \epsilon i-\epsilon^{2}\right) \int_{B_{2 \eta}(y)} u_{\epsilon}(z, x) n(z)(1-\chi(|z-y|)) G(z, y) d z \\
& +G_{n, \epsilon}(x, y)-\left(2 k \epsilon i-\epsilon^{2}\right) \int_{B_{2 \eta}(x)} u_{\epsilon}(z, y) n(z)(1-\chi(|z-x|)) G(z, x) d z \\
& =\int_{B_{r}(0) \cap \mathbb{R}_{+}^{2}} G_{n, \epsilon}(z, x)\left[\Delta_{z}+(k+i \epsilon)^{2} n(z)\right] G_{n, \epsilon}(z, y) d z \\
& -\int_{B_{r}(0) \cap \mathbb{R}_{+}^{2}} G_{n, \epsilon}(z, y)\left[\Delta_{z}+(k+i \epsilon)^{2} n(z)\right] G_{n, \epsilon}(z, x) d z \\
& =\int_{\partial B_{r}(0) \cap \mathbb{R}_{+}^{2}} u_{\epsilon}(z, x) \frac{\partial u_{\epsilon}(z, y)}{\partial \nu_{z}}-u_{\epsilon}(z, y) \frac{\partial u_{\epsilon}(z, x)}{\partial \nu_{z}} d s(z) .
\end{aligned}
$$

Since $u_{\epsilon} \in H^{1}\left(\mathbb{R}_{+}^{2}\right)$, the right hand side of 4.9 converges as $r \rightarrow \infty$ to zero. Then, as $r \rightarrow \infty$ in 4.9 we have

$$
\begin{aligned}
& G_{n, \epsilon}(x, y)-G_{n, \epsilon}(y, x) \\
& \quad=\left(2 k \epsilon i-\epsilon^{2}\right) \int_{B_{2 \eta}(x)} u_{\epsilon}(z, y) n(z)(1-\chi(|z-x|)) G(z, x) d z \\
& \quad-\left(2 k \epsilon i-\epsilon^{2}\right) \int_{B_{2 \eta}(y)} u_{\epsilon}(z, x) n(z)(1-\chi(|z-y|)) G(z, y) d z
\end{aligned}
$$

Since $u_{\epsilon}$ converges as $\epsilon \rightarrow+0$ in $H_{l o c}^{1}\left(\mathbb{R}_{+}^{2}\right)$ to $u$, the right hand side of (4.10) converges to zero as $\epsilon \rightarrow+0$. Therefore, we conclude that $G_{n}(x, y)=$ $G_{n}(y, x)$ for $x \neq y$.

By the symmetricity of $G_{n}$,

$$
\begin{aligned}
& \langle H g, f\rangle=\int_{Q}\left\{\sqrt{|n(x) q(x)|} \int_{M} \overline{G_{n}(x, y)} g(y) d s(y)\right\} \overline{f(x)} d x
\end{aligned}
$$

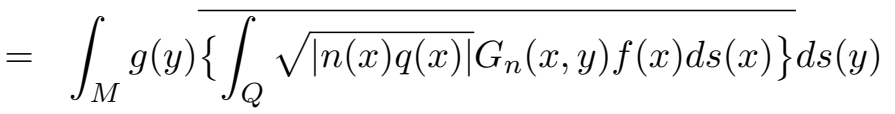

$$
\begin{aligned}
& =\int_{M} g(y) \overline{\left\{\int_{Q} \sqrt{|n(x) q(x)|} G_{n}(y, x) f(x) d s(x)\right\}} d s(y),
\end{aligned}
$$

which implies that

$$
H^{*} f(x)=\int_{Q} \sqrt{|n(y) q(y)|} G_{n}(x, y) f(y) d s(y), x \in M .
$$


We define $T: L^{2}(Q) \rightarrow L^{2}(Q)$ by $T f:=\frac{|n q|}{k^{2} n q} f-\sqrt{|n q|} w$ where $w$ satisfies the radiation condition and

$$
\begin{gathered}
\Delta w+k^{2} n w=-\sqrt{|n q|} f, \text { in } \mathbb{R}_{+}^{2}, \\
v=0 \text { on } \mathbb{R} \times\{0\} .
\end{gathered}
$$

We will show the following integral representation of $w$.

\section{Lemma 4.2 .}

$$
w(x)=\int_{Q} \sqrt{|n(y) q(y)|} G_{n}(x, y) f(y) d y, x \in \mathbb{R}_{+}^{2} .
$$

Proof of Lemma 4.2. Let $w_{\epsilon} \in H_{l o c}^{1}\left(\mathbb{R}_{+}^{2}\right)$ be a solution of the problem (4.13) -4.14) replacing $k$ by $(k+i \epsilon)$ satisfying $w_{\epsilon}$ converges as $\epsilon \rightarrow+0$ in $H_{l o c}^{1}\left(\mathbb{R}_{+}^{2}\right)$ to $w$. Let $G_{n, \epsilon}(y, x)$ be an approximation of the Green's function $G_{n}(y, x)$ as same as in Lemma 4.1. Let $r>0$ be large enough such that $x \in B_{r}(0)$. By Green's second theorem in $B_{r}(0) \cap \mathbb{R}_{+}^{2}$ we have

$$
\begin{aligned}
- & w_{\epsilon}(x)+\left(2 k \epsilon i-\epsilon^{2}\right) \int_{B_{2 \eta}(x)} w_{\epsilon}(y) n(y)(1-\chi(|y-x|)) G(y, x) d y \\
+ & \int_{Q} \sqrt{|n(y) q(y)|} G_{n, \epsilon}(y, x) f(y) d y \\
= & \int_{B_{r}(0) \cap \mathbb{R}_{+}^{2}} w_{\epsilon}(y)\left[\Delta_{y}+(k+i \epsilon)^{2} n(y)\right] G_{n, \epsilon}(y, x) d y \\
- & \int_{B_{r}(0) \cap \mathbb{R}_{+}^{2}} G_{n, \epsilon}(y, x)\left[\Delta_{y}+(k+i \epsilon)^{2} n(y)\right] w_{\epsilon}(y) d z \\
= & \int_{\partial B_{r}(0) \cap \mathbb{R}_{+}^{2}} w_{\epsilon}(y) \frac{\partial u_{\epsilon}(y, x)}{\partial \nu_{y}}-u_{\epsilon}(y, x) \frac{\partial w_{\epsilon}(y)}{\partial \nu_{y}} d s(y) .
\end{aligned}
$$

Since $u_{\epsilon}, w_{\epsilon} \in H^{1}\left(\mathbb{R}_{+}^{2}\right)$, the right hand side of 4.16 converges as $r \rightarrow \infty$ to zero. Then, as $r \rightarrow \infty$ in 4.16 ) we have

$$
\begin{aligned}
w_{\epsilon}(x) & =\left(2 k \epsilon i-\epsilon^{2}\right) \int_{B_{2 \eta}(x)} w_{\epsilon}(y) n(y)(1-\chi(|y-x|)) G(y, x) d y \\
& +\int_{Q} \sqrt{|n(y) q(y)|} G_{n, \epsilon}(y, x) f(y) d y
\end{aligned}
$$

The first term of right hand side in (4.17) converges to zero as $\epsilon \rightarrow+0$, and the second term converges to $\int_{Q} \sqrt{|n(y) q(y)|} G_{n}(y, x) f(y) d y$ as $\epsilon \rightarrow+0$. As $\epsilon \rightarrow+0$ in 4.17 and by the symmetricity of $G_{n}$ (Lemma 4.1) we conclude (4.15). 
Since $w$ satisfies

$$
\begin{aligned}
\Delta w+k^{2}(1+q) n w & =-k^{2} \frac{n q}{\sqrt{|n q|}}\left\{\frac{|n q|}{k^{2} n q} f-\sqrt{|n q|} w\right\} \text { in } \mathbb{R}_{+}^{2} \\
& =-k^{2} \frac{n q}{\sqrt{|n q|}} T f,
\end{aligned}
$$

we have $\left.w\right|_{M}=L T f$. Therefore, by 4.12 and 4.15 we have $H^{*}=L T$. Then, we have the following symmetric factorization:

$$
N=L T^{*} L^{*} \text {. }
$$

We will show the following lemma corresponding to the assumptions in Theorem 3.1.

Lemma 4.3. (a) $L$ is compact with dense range in $L^{2}(M)$.

(b) If there exists the constant $q_{\min }>0$ such that $q_{\min } \leq q$ a.e. in $Q$, then $\operatorname{Re} T$ has the form $\operatorname{Re} T=C+K$ with some self-adjoint and positive coercive operator $C$ and some compact operator $K$ on $L^{2}(Q)$.

(c) $\operatorname{Im}\langle f, T f\rangle \geq 0$ for all $f \in L^{2}(Q)$.

(d) $T$ is injective.

Proof of Lemma 4.3. (d) Let $f \in L^{2}(Q)$ and $T f=0$, i.e., $\frac{|n q|}{k^{2} n q} f=$ $\sqrt{|n q|} w$ where $w$ satisfies $4.13-4.14$. Then, $\Delta w+k^{2} n(1+q) w=0$. Вy the uniqueness, $w=0$ in $\mathbb{R}_{+}^{2}$ which implies that $f=0$. Therefore $T$ is injective.

(b) Since $n$ and $q$ are bounded below (that is, $n \geq n_{\min }>0$ and $\left.q \geq q_{\text {min }}>0\right), T$ has the form $T=C+K$ where $K$ is some compact operator and $C$ is some self-adjoint and positive coercive operator. Furthermore, from the injectivity of $T$ we obtain that $T$ is bijective.

(a) By the trace theorem and $v \in H_{l o c}^{1}\left(\mathbb{R}_{+}^{2}\right), L f=\left.v\right|_{M} \in H^{1 / 2}(M)$, which implies that $L: L^{2}(Q) \rightarrow L^{2}(M)$ is compact.

By the bijectivity of $T$ and $H=T^{*} L^{*}$, it is sufficient to show the injectivity of $H$. Let $g \in L^{2}(M)$ and $H g(x)=\sqrt{|n(x) q(x)|} \int_{M} \overline{G_{n}(x, y)} g(y) d s(y)=$ 0 for $x \in Q$. We set $v(x):=\int_{M} \overline{G_{n}(x, y)} g(y) d s(y)$. By the definition of $v$ we have

$$
\Delta v+k^{2} n v=0, \text { in } \mathbb{R}_{+}^{2} \backslash M,
$$

and since $q$ are bounded below, $v=0$ in $Q$. By unique continuation principle we have $v=0$ in $\mathbb{R}_{+}^{2} \backslash M$. By the jump relation (see [15]) we have $0=$ $\frac{\partial v_{+}}{\partial \nu}-\frac{\partial v_{-}}{\partial \nu}=g$, which conclude that the operator $H$ is injective. 
(c) For the proof of (c) we refer to Theorem 3.1 in [1]. By the definition of $T$ we have

$$
\operatorname{Im}\langle f, T f\rangle=-\operatorname{Im} \int_{Q} f \sqrt{|n q|} \bar{w} d x=\operatorname{Im} \int_{Q} \bar{w}\left[\Delta+k^{2} n\right] w d x
$$

where $w$ is a radiating solution of the problem (4.13)-(4.14). We set $\Omega_{N}:=$ $(-N, N) \times\left(0, N^{s}\right)$ where $s>0$ is small enough and $N>0$ is large enough. By the same argument in Theorem 3.1 of [1] we have

$$
\begin{aligned}
& \operatorname{Im}\langle f, T f\rangle=\operatorname{Im} \int_{\Omega_{N}} \bar{w}\left[\Delta+k^{2} n\right] w d x=\operatorname{Im} \int_{\Omega_{N}} \bar{w} \Delta w d x \\
& \geq\left[\frac{1}{2 \pi} \sum_{j \in J} \sum_{d_{l, j}, d_{l^{\prime}, j}>0} \overline{a_{l, j}} a_{l^{\prime}, j} \int_{C_{\phi(N)}} \overline{\phi_{l, j}} \frac{\partial \phi_{l^{\prime}, j}}{\partial x_{1}} d x\right] \\
& -\operatorname{Im}\left[\frac{1}{2 \pi} \sum_{j \in J} \sum_{l_{l, j}, d_{l^{\prime}, j}<0} \overline{a_{l, j}} a_{l^{\prime}, j} \int_{C_{\phi(N)}} \overline{\phi_{l, j}} \frac{\partial \phi_{l^{\prime}, j}}{\partial x_{1}} d x\right]+o(1),
\end{aligned}
$$

where where some $a_{l, j} \in \mathbb{C}$, and $\left\{d_{l, j}, \phi_{l, j}: l=1, \ldots, m_{j}\right\}$ are normalized eigenvalues and eigenfunctions of the problem (2.8). By Lemmas 6.3 and 6.4 of [11, as $N \rightarrow \infty$ in 4.22 we have

$$
\operatorname{Im}\langle f, T f\rangle \geq \frac{k}{2 \pi} \sum_{j \in J}\left[\sum_{d_{l, j}>0}\left|a_{l, j}\right|^{2} d_{l, j}-\sum_{d_{l, j}<0}\left|a_{l, j}\right|^{2} d_{l, j}\right] \geq 0,
$$

which concludes (c).

Remark 4.4. The strictly positivity of $\operatorname{Im} T$ is missing in Lemma 4.3 although we have the injectivity of $T$. From the viewpoint of Section 3, the assumption of transmission eigenvalue of $Q$ can be expected when we apply Theorem 3.1 to this case. However, even with its assumption the author of this paper do not have the idea to show $\operatorname{Im} T>0$.

In order to show Theorems 1.1 and 1.2, we consider another factorization of the near field operator $N$. We define $\tilde{T}: L^{2}(Q) \rightarrow L^{2}(Q)$ by $\tilde{T} v:=$ $k^{2} \frac{n q}{|n q|} g-k^{2} \frac{n q}{\sqrt{|n q|}} v$ where $v$ satisfies the radiation condition and

$$
\begin{gathered}
\Delta v+k^{2}(1+q) n v=-k^{2} \frac{n q}{\sqrt{|n q|}} g, \text { in } \mathbb{R}_{+}^{2}, \\
v=0 \text { on } \mathbb{R} \times\{0\} .
\end{gathered}
$$


Then, by the definition of $T$ and $\tilde{T}$ we can show that $\tilde{T} T=I$ and $T \tilde{T}=I$, which implies that $T^{-1}=\tilde{T}$. Therefore, we have by $L=H^{*} T^{-1}$

$$
N=L T^{*} L^{*}=H^{*} T^{-1} H=H^{*} \tilde{T} H=H_{Q}^{*} \hat{T} H_{Q},
$$

where $H_{Q}: L^{2}(M) \rightarrow L^{2}(Q)$ is defined by

$$
H_{Q} g(x):=\int_{M} \overline{G_{n}(x, y)} g(y) d s(y), x \in Q,
$$

and $\hat{T}: L^{2}(Q) \rightarrow L^{2}(Q)$ is defined by $\hat{T} f=k^{2} n q f+k^{2} n q w$ where $w$ satisfies the radiation condition and

$$
\begin{gathered}
\Delta w+k^{2}(1+q) n w=-k^{2} n q f, \text { in } \mathbb{R}_{+}^{2}, \\
w=0 \text { on } \mathbb{R} \times\{0\} .
\end{gathered}
$$

We will show the following lemma.

Lemma 4.5. Let $B$ and $Q$ be a bounded open set in $\mathbb{R}_{+}^{2}$.

(a) $\operatorname{dim}\left(\operatorname{Ran}\left(H_{B}^{*}\right)\right)=\infty$.

(b) If $B \cap Q=\emptyset$, then $\operatorname{Ran}\left(H_{B}^{*}\right) \cap \operatorname{Ran}\left(H_{Q}^{*}\right)=\{0\}$.

Proof of Lemma 4.4. (a) By the same argument of the injectivity of $H$ in (a) of Lemma 4.3 , we can show that $H_{B}$ is injective. Therefore, $H_{B}^{*}$ has dense range.

(b) Let $h \in \operatorname{Ran}\left(H_{B}^{*}\right) \cap \operatorname{Ran}\left(H_{Q}^{*}\right)$. Then, there exists $f_{B}$, $f_{Q}$ suct that $h=H_{B}^{*} f_{B}=H_{Q}^{*} f_{Q}$. We set

$$
\begin{aligned}
& v_{B}(x):=\int_{B} G_{n}(x, y) f_{B}(y) d y, x \in \mathbb{R}_{+}^{2} \\
& v_{Q}(x):=\int_{Q} G_{n}(x, y) f_{Q}(y) d y, x \in \mathbb{R}_{+}^{2}
\end{aligned}
$$

then, $v_{B}$ and $v_{Q}$ satisfies $\Delta v_{B}+k^{2} n v_{B}=-f_{B}$, and $\Delta v_{Q}+k^{2} n v_{Q}=-f_{Q}$, respectivelty, and $v_{B}=v_{Q}$ on $M$. By Rellich lemma and unique continuation we have $v_{B}=v_{Q}$ in $\mathbb{R}_{+}^{2} \backslash(\overline{B \cap Q})$. Hence, we can define $v \in H_{l o c}^{1}\left(\mathbb{R}^{2}\right)$ by

$$
v:= \begin{cases}v_{B}=v_{Q} & \text { in } \mathbb{R}_{+}^{2} \backslash(\overline{B \cap Q}) \\ v_{B} & \text { in } Q \\ v_{Q} & \text { in } B\end{cases}
$$

and $v$ is a radiating solution such that $v=0$ for $x_{2}=0$ and

$$
\Delta v+k^{2} n v=0 \text { in } \mathbb{R}_{+}^{2} .
$$

By the uniquness, we have $v=0$ in $\mathbb{R}^{2}$, which implies that $h=0$. 
In the following sections we will show Theorems 1.1 and 1.2 by using properties of the factorization of the near field operator $N$.

\section{$5 \quad$ Proof of Theorem 1.1}

In Section 5, we will show Theorem 1.1. Let $B \subset Q$. We define $K$ : $L^{2}(Q) \rightarrow L^{2}(Q)$ by $K f:=k^{2} n q w$ where $w$ is a radiating solution of the problem 4.28- 4.29). Since $\left.w\right|_{Q} \in H^{1}(Q), K$ is a compact operator. Let $V$ be the sum of eigenspaces of $\operatorname{Re} K$ associated to eigenvalues less than $\alpha-k^{2} n_{\min } q_{\min }$. Since $\alpha-k^{2} n_{\min } q_{\min }<0$, then $V$ is a finite dimensional and for $H_{Q} g \in V^{\perp}$

$$
\begin{aligned}
\langle\operatorname{Re} N g, g\rangle & =\int_{Q} k^{2} n q\left|H_{Q} g\right|^{2} d x+\left\langle(\operatorname{Re} K) H_{Q} g, H_{Q} g\right\rangle \\
& \geq k^{2} n_{\text {min }} q_{\text {min }}\left\|H_{Q} g\right\|^{2}+\left(\alpha-k^{2} n_{\text {min }} q_{\text {min }}\right)\left\|H_{Q} g\right\|^{2} \\
& \geq \alpha\left\|H_{Q} g\right\|^{2} \geq \alpha\left\|H_{B} g\right\|^{2}
\end{aligned}
$$

Since for $g \in L^{2}(M)$

$$
H_{Q} g \in V^{\perp} \Longleftrightarrow g \in\left(H_{Q}^{*} V\right)^{\perp},
$$

and $\operatorname{dim}\left(H_{Q}^{*} V\right) \leq \operatorname{dim}(V)<\infty$, we have by Corollary 3.3 of [5] that $\alpha H_{B}^{*} H_{B} \leq_{\text {fin }} \operatorname{Re} N$.

Let now $B \not \subset Q$ and assume on the contrary $\alpha H_{B}^{*} H_{B} \leq_{\text {fin }} \operatorname{Re} N$, that is, by Colrollary 3.3 of [5] there exists a finite dimensional subspace $W$ in $L^{2}(M)$ such that

$$
\left\langle\left(\operatorname{Re} N-\alpha H_{B}^{*} H_{B}\right) w, w\right\rangle \geq 0,
$$

for all $w \in W^{\perp}$. Since $B \not \subset Q$, we can take a small open domain $B_{0} \subset B$ such that $B_{0} \cap Q=\emptyset$, which implies that for all $w \in W^{\perp}$

$$
\begin{aligned}
\alpha\left\|H_{B_{0}} w\right\|^{2} & \leq \alpha\left\|H_{B} w\right\|^{2} \\
& \leq\langle(\operatorname{Re} N) w, w\rangle \\
& =\left\langle(\operatorname{Re} \hat{T}) H_{Q} w, H_{Q} w\right\rangle \\
& \leq\|\operatorname{Re} \hat{T}\|\left\|H_{Q} w\right\|^{2} .
\end{aligned}
$$

By (a) of Lemma 4.7 in [5], we have

$$
\operatorname{Ran}\left(H_{B_{0}}^{*}\right) \nsubseteq \operatorname{Ran}\left(H_{Q}^{*}\right)+W=\operatorname{Ran}\left(H_{Q}^{*}, P_{W}\right),
$$


where $P_{W}: L^{2}(M) \rightarrow L^{2}(M)$ is the orthogonal projection on $W$. Lemma 4.6 of [5] implies that for any $C>0$ there exists a $w_{c}$ such that

$$
\left\|H_{B_{0}} w_{c}\right\|^{2}>C^{2}\left\|\left(\begin{array}{c}
H_{Q} \\
P_{V}
\end{array}\right) w_{c}\right\|^{2}=C^{2}\left(\left\|H_{Q} w_{c}\right\|^{2}+\left\|P_{W} w_{c}\right\|^{2}\right) .
$$

Hence, there exists a sequence $\left(w_{m}\right)_{m \in \mathbb{N}} \subset L^{2}\left(\mathbb{S}^{1}\right)$ such that $\left\|H_{B_{0}} w_{m}\right\| \rightarrow \infty$ and $\left\|H_{Q} w_{m}\right\|+\left\|P_{V} w_{m}\right\| \rightarrow 0$ as $m \rightarrow \infty$. Setting $\tilde{w}_{m}:=w_{m}-P_{W} w_{m} \in W^{\perp}$ we have as $m \rightarrow \infty$,

$$
\begin{gathered}
\left\|H_{B_{0}} \tilde{w}_{m}\right\| \geq\left\|H_{B_{0}} w_{m}\right\|-\left\|H_{B_{0}}\right\|\left\|P_{W} w_{m}\right\| \rightarrow \infty, \\
\left\|H_{Q} \tilde{w}_{m}\right\| \leq\left\|H_{Q} w_{m}\right\|+\left\|H_{Q}\right\|\left\|P_{W} w_{m}\right\| \rightarrow 0 .
\end{gathered}
$$

This contradicts (5.4). Therefore, we have $\alpha H_{B}^{*} H_{B} \not_{\text {fin }} \operatorname{Re} N$. Theorem 1.1 has been shown.

By the same argument in Theorem 1.1 we can show the following.

Corollary 5.1. Let $B \subset \mathbb{R}^{2}$ be a bounded open set. Let Assumption hold, and assume that there exists $q_{\max }<0$ such that $q \leq q_{\max }$ a.e. in $Q$. Then for $0<\alpha<k^{2} n_{\min }\left|q_{\max }\right|$,

$$
B \subset Q \quad \Longleftrightarrow \quad \alpha H_{B}^{*} H_{B} \leq_{\text {fin }}-\operatorname{Re} N
$$

\section{Proof of Theorem 1.2}

In Section 6, we will show Theorem 1.2. Let $Q \subset B$. Let $V$ be the sum of eigenspaces of $\operatorname{Re} K$ associated to eigenvalues more than $\alpha-k^{2} n_{\max } q_{\max }$. Since $\alpha-k^{2} n_{\max } q_{\max }>0$, then $V$ is a finite dimensional and for $H_{Q} g \in V^{\perp}$

$$
\begin{aligned}
\langle\operatorname{Re} N g, g\rangle & =\int_{Q} k^{2} n q\left|H_{Q} g\right|^{2} d x+\left\langle(\operatorname{Re} K) H_{Q} g, H_{Q} g\right\rangle \\
& \leq k_{\text {max }}^{n} q_{\max }\left\|H_{Q} g\right\|^{2}+\left(\alpha-k^{2} n_{\max } q_{\max }\right)\left\|H_{Q} g\right\|^{2} \\
& \leq \alpha\left\|H_{Q} g\right\|^{2} \leq \alpha\left\|H_{B} g\right\|^{2} .
\end{aligned}
$$

Since for $g \in L^{2}(M)$

$$
H_{Q} g \in V^{\perp} \quad \Longleftrightarrow \quad g \in\left(H_{Q}^{*} V\right)^{\perp}
$$

and $\operatorname{dim}\left(H_{Q}^{*} V\right) \leq \operatorname{dim}(V)<\infty$, we have by Corollary 3.3 of [5] that $\operatorname{Re} N \leq_{\text {fin }} \alpha H_{B}^{*} H_{B}$ 
Let now $Q \not \subset B$ and assume on the contrary $\operatorname{Re} N \leq_{\text {fin }} \alpha H_{B}^{*} H_{B}$, that is, by Corollary 3.3 of $[5$ there exists a finite dimensional subspace $W$ in $L^{2}(M)$ such that

$$
\left\langle\left(\alpha H_{B}^{*} H_{B}-\operatorname{Re} N\right) w, w\right\rangle \geq 0,
$$

for all $w \in W^{\perp}$. Since $Q \not \subset B$, we can take a small open domain $Q_{0} \subset Q$ such that $Q_{0} \cap B=\emptyset$. Let $V$ be the sum of eigenspaces of Re $K$ associated to eigenvalues less than $-k^{2} n_{\min } q_{\min } / 2$. Then, $V$ is a finite dimensional and for $g \in\left(H_{Q}^{*} V\right)^{\perp} \cap W^{\perp}=\left(H_{Q}^{*} V \cup W\right)^{\perp}$ we have

$$
\begin{aligned}
& \alpha\left\|H_{B} g\right\|^{2} \\
\geq & \langle(\operatorname{Re} N) g, g\rangle \\
= & \int_{Q} k^{2} n q\left|H_{Q} g\right|^{2} d x+\left\langle(\operatorname{Re} \hat{K}) H_{Q} g, H_{Q} g\right\rangle \\
\geq & k^{2} n_{\min } q_{\min }\left\|H_{Q} g\right\|^{2}-k^{2} n_{\min } q_{\min } / 2\left\|H_{Q} g\right\|^{2}=k^{2} n_{\min } q_{\min } / 2\left\|H_{Q} g\right\|^{2} \\
\geq & k^{2} n_{\min } q_{\min } / 2\left\|H_{Q_{0}} g\right\|^{2},
\end{aligned}
$$

and $\operatorname{dim}\left(H_{Q}^{*} V \cup W\right)<\infty$. By the same argument in Theorem 1.1, there exists a sequence $\left(g_{m}\right)_{m \in \mathbb{N}} \subset\left(H_{Q}^{*} V \cup W\right)^{\perp}$ such that $\left\|H_{Q_{0}} w_{m}\right\| \rightarrow \infty$ and $\left\|H_{B} g_{m}\right\| \rightarrow 0$ as $m \rightarrow \infty$, which contradicts (6.4). Therefore, we have $\operatorname{Re} N \mathbb{f}_{\text {fin }} \alpha H_{B}^{*} H_{B}$. Theorem 1.2 has been shown.

By the same argument in Theorem 1.2 we can show the following.

Corollary 6.1. Let $B \subset \mathbb{R}^{2}$ be a bounded open set. Let Assumption hold, and assume that there exists $q_{\min }<0$ and $q_{\max }<0$ such that $q_{\min } \leq q \leq$ $q_{\max }$ a.e. in $Q$. Then for $\alpha>k^{2} n_{\max }\left|q_{\min }\right|$,

$$
Q \subset B \quad \Longleftrightarrow \quad-\operatorname{Re} N \leq_{\text {fin }} \alpha H_{B}^{*} H_{B}
$$

\section{Numerical examples}

In Section 7, we discuss the numerical examples based on Theorem 1.1. We consider the following two supports $Q_{1}$ and $Q_{2}$ of functions $q_{1}, q_{2}$ (see Figure 1):

(1) $Q_{1}=\left\{\left(x_{1}, x_{2}\right) \mid\left(x_{1}-0.5\right)^{2}+\left(x_{2}-0.5\right)^{2}<(0.2)^{2}\right\}$

(2) $Q_{2}=\left\{\left(x_{1}, x_{2}\right) \mid\left(\left(x_{1}-0.5\right) / 0.15\right)^{2}+\left(\left(x_{2}-0.6\right) / 0.3\right)^{2}<1\right\}$ 
where $q_{1}$ and $q_{2}$ are defined by

$$
q_{j}(x):= \begin{cases}1 & \text { for } x \in Q_{j} \\ 0 & \text { for } x \notin Q_{j}\end{cases}
$$

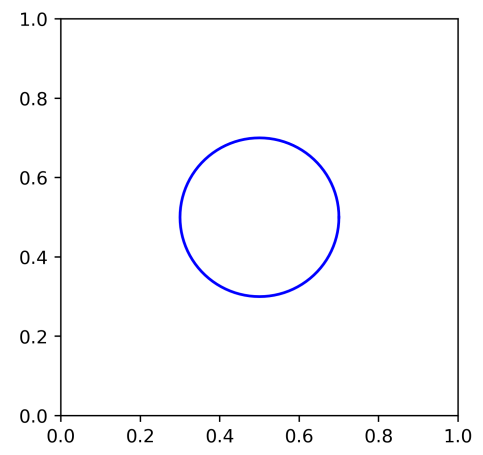

$Q_{1}$

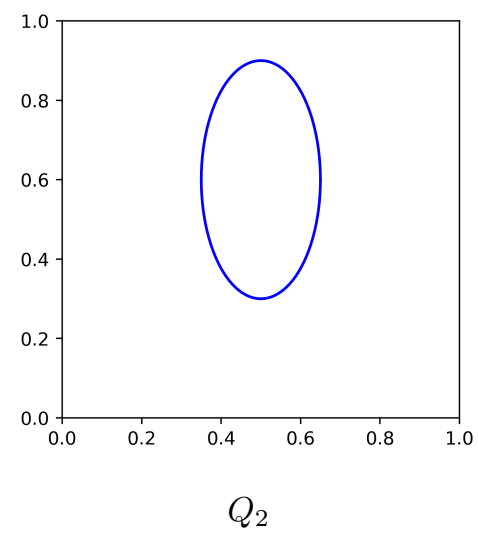

Figure 1: The original shape

Based on Theorem 1.1, the indicator function in our examples is given by

$$
I(B):=\#\left\{\text { negative eigenvalues of } \operatorname{Re} N-\alpha H_{B}^{*} H_{B}\right\}
$$

The idea to recover $Q_{j}$ is to plot the value of $I(B)$ for many of small $B$ in the certain sampling region. Then, we expect from Theorem 1.1 that the value of the function $I(\sigma)$ is low if $B$ is included in $Q_{j}$.

We consider the sampling region by $[0, R] \times[0, R]$ with some $R>0$. The test domain $B$ is given by the small square $B_{i, j}:=z_{i, j}+[-R / 2 M, R / 2 M]^{2}$ where the location $z_{i, j}=(R i / M, R j / M)(i, j=1, \ldots, M)$ and $M$ is some large number.

The near field operator $N$ is discretized by the matrix

$$
N \approx \frac{b-a}{d}\left(u^{s}\left(x_{l}, x_{p}\right)\right)_{1 \leq l, p \leq d} \in \mathbb{C}^{d \times d}
$$

where $\hat{x}_{l}=\left(a+\frac{(b-a) l}{d}, m\right)$, and the scattered field $u^{s}$ of the problem 1.1 (1.2) is a solution of the following integral equation

$u^{s}(x, z)=k^{2} \int_{Q} q(y) n(y) u^{s}(y, z) G_{n}(x, y) d y+k^{2} \int_{Q} q(y) n(y) \overline{G_{n}(y, z)} G_{n}(x, y) d y$. 
In our examples we fix $R=1, M=100, d=30, a=-25, b=25, m=20$, and $n \equiv 1$. Figure 2 is given by plotting the values of the indicator function

$$
I_{\text {square }}\left(z_{i, j}\right):=I\left(B_{i, j}\right), i, j=1, \ldots, 100,
$$

for two different supports $Q_{1}$ and $Q_{2}$ of true functions $q_{1}$ and $q_{2}$, and for two different parameters $\alpha=10,20$ in the case of wavenumber $k=5$.
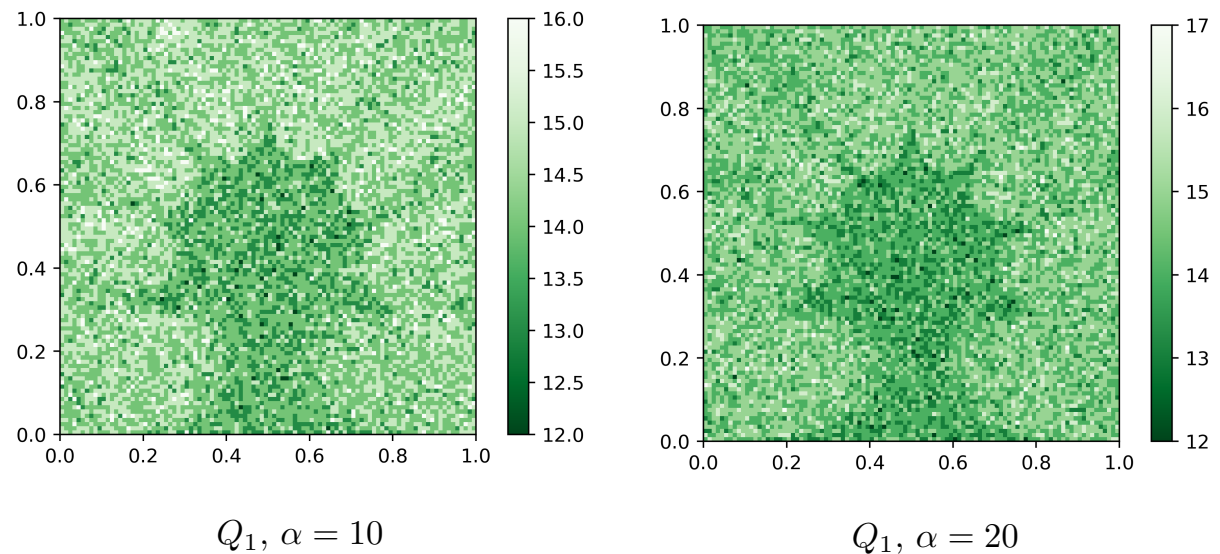

$Q_{1}, \alpha=20$
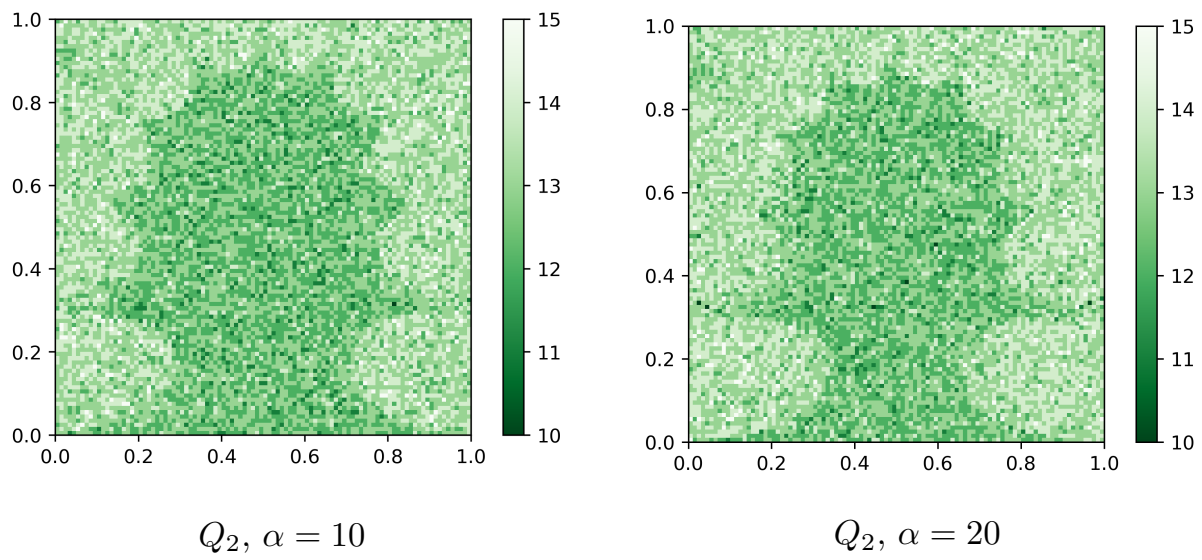

Figure 2: Reconstruction by the indicator function $I_{\text {square }}$ in the case of wavenumber $k=5$ 


\section{Acknowledgments}

The author thanks to Professor Andreas Kirsch, who supports him in this study.

\section{References}

[1] T. Furuya, Scattering by the local perturbation of an open periodic waveguide in the half plane, preprint arXiv:1906.01180, (2019).

[2] T. Furuya, T. Daimon, R. Saiin, The monotonicity method for the inverse crack scattering problem, to appear in Inverse Probl. Sci. Eng., $(2020)$.

[3] R. Griesmaier, B. Harrach, Monotonicity in inverse medium scattering on unbounded domains, SIAM J. Appl. Math. 78, (2018), no. 5, 25332557.

[4] B. Harrach, V. Pohjola, M. Salo, Dimension bounds in monotonicity methods for the Helmholtz equation, SIAM J. Appl. Math. 51, (2019), no. 4, 2995-3019.

[5] B. Harrach, V. Pohjola, M. Salo, Monotonicity and local uniqueness for the Helmholtz equation, Anal. PDE, 12, (2019), no. 7, 1741-1771.

[6] B. Harrach, M. Ullrich, Local uniqueness for an inverse boundary value problem with partial data, Proc. Amer. Math. Soc. 145, (2017), no. 3, 1087-1095.

[7] B. Harrach, M. Ullrich, Monotonicity based shape reconstruction in electrical impedance tomography, SIAM J. Math. Anal. 45, (2013), no. 6, 3382-3403.

[8] A. Kirsch, The factorization method for a class of inverse elliptic problems, Math. Nachr. 278, (2004), 258-277.

[9] A. Kirsch and N. Grinberg, The factorization method for inverse problems, Oxford University Press, (2008).

[10] A. Kirsch, A. Lechleiter, A radiation condition arising from the limiting absorption principle for a closed full- or half-waveguide problem, Math. Methods Appl. Sci. 41, (2018), no. 10, 3955-3975. 
[11] A. Kirsch, A. Lechleiter, The limiting absorption principle and a radiation condition for the scattering by a periodic layer, SIAM J. Math. Anal. 50 (2018), no. 3, 2536-2565.

[12] E. Lakshtanov, A. Lechleiter, Difference factorizations and monotonicity in inverse medium scattering for contrasts with fixed sign on the boundary, SIAM J. Math. Anal. 48 (2016), no. 6, 3688-3707.

[13] A. Lechleiter, The factorization method is independent of transmission eigenvalues, Inverse Probl. Imaging 3 (2009), 123-138.

[14] A. Lechleiter, The Floquet-Bloch transform and scattering from locally perturbed periodic surfaces, J. Math. Anal. Appl. 446, (2017), no. 1, 605-627.

[15] W. McLean, Strongly elliptic systems and boundary integral equations, Cambridge University Press, Cambridge, (2000).

Graduate School of Mathematics, Nagoya University, Furocho, Chikusaku, Nagoya, 464-8602, Japan

e-mail: takashi.furuya0101@gmail.com 